\title{
stellaR: A Package to Manage Stellar Evolution Tracks and Isochrones
}

by Matteo Dell'Omodarme and Giada Valle

\begin{abstract}
We present the R package stellaR, which is designed to access and manipulate publicly available stellar evolutionary tracks and isochrones from the Pisa low-mass database. The procedures for extracting important stages in the evolution of a star from the database, for constructing isochrones from stellar tracks and for interpolating among tracks are discussed and demonstrated.
\end{abstract}

Due to the advance in the instrumentation, nowadays astronomers can deal with a huge amount of high-quality observational data. In the last decade impressive improvements of spectroscopic and photometric observational capabilities made available data which stimulated the research in the globular clusters field. The theoretical effort of recovering the evolutionary history of the clusters benefits from the computation of extensive databases of stellar tracks and isochrones, such as Pietrinferni et al. (2006); Dotter et al. (2008); Bertelli et al. (2008). We recently computed a large data set of stellar tracks and isochrones, "The Pisa low-mass database" (Dell'Omodarme et al., 2012), with up to date physical and chemical inputs, and made available all the calculations to the astrophysical community at the Centre de Données astronomiques de Strasbourg $(\mathrm{CDS})^{1}$, a data center dedicated to the collection and worldwide distribution of astronomical data.

In most databases, the management of the information and the extraction of the relevant evolutionary properties from libraries of tracks and/or isochrones is the responsibility of the end users. Due to its extensive capabilities of data manipulation and analysis, however, $\mathrm{R}$ is an ideal choice for these tasks. Nevertheless R is not yet well known in astrophysics; up to December 2012 only seven astronomical or astrophysical-oriented packages have been published on CRAN (see the CRAN Task View Chemometrics and Computational Physics).

The package stellaR (Dell'Omodarme and Valle, 2012) is an effort to make available to the astrophysical community a basic tool set with the following capabilities: retrieve the required calculations from CDS; plot the information in a suitable form; construct by interpolation tracks or isochrones of compositions different to the ones available in the database; construct isochrones for age not included in the database; extract relevant evolutionary points from tracks or isochrones.

\section{Get stellar evolutionary data}

The Pisa low-mass database contains computations classified according to four parameters: the metallicity $z$ of the star, its initial helium value $y$, the value of $\alpha$-enhancement of the heavy elements mixture with respect to the reference mixture and the mixing-length parameter $\alpha_{\mathrm{ml}}$ used to model external convection efficiency. The values of the parameters available in the database can be displayed using the function showComposition():

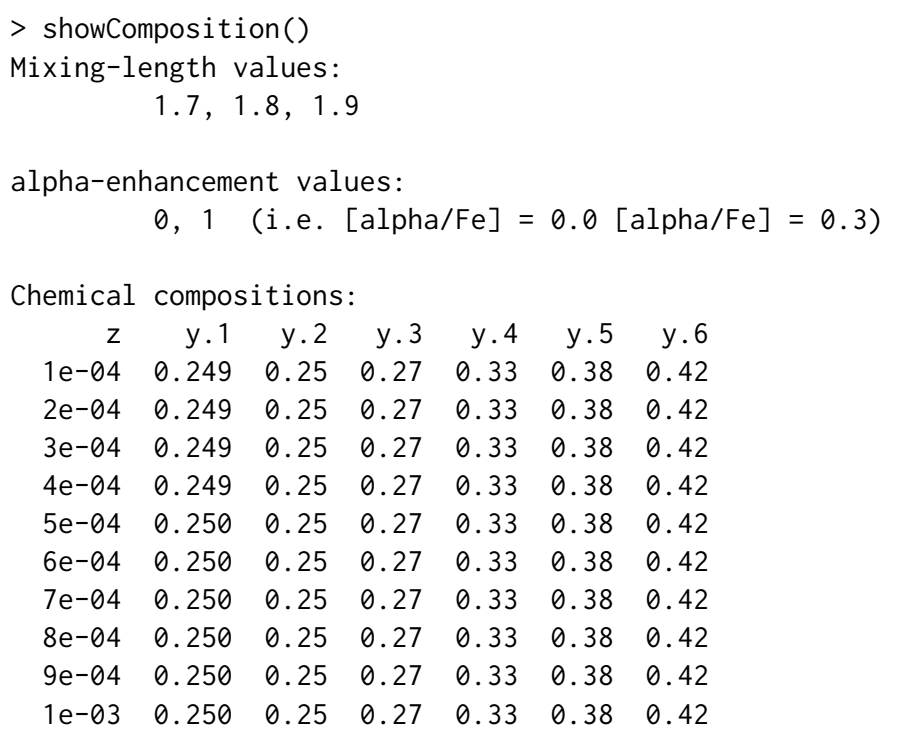

\footnotetext{
${ }^{1}$ via anonymous ftp from ftp://cdsarc.u-strasbg. fr or via http://cdsarc.u-strasbg. fr/viz-bin/qcat?J/ A+A/540/A26
} 


$\begin{array}{lllllll}2 \mathrm{e}-03 & 0.252 & 0.25 & 0.27 & 0.33 & 0.38 & 0.42 \\ 3 \mathrm{e}-03 & 0.254 & 0.25 & 0.27 & 0.33 & 0.38 & 0.42 \\ 4 \mathrm{e}-03 & 0.256 & 0.25 & 0.27 & 0.33 & 0.38 & 0.42 \\ 5 \mathrm{e}-03 & 0.258 & 0.25 & 0.27 & 0.33 & 0.38 & 0.42 \\ 6 \mathrm{e}-03 & 0.260 & 0.25 & 0.27 & 0.33 & 0.38 & 0.42 \\ 7 \mathrm{e}-03 & 0.262 & 0.25 & 0.27 & 0.33 & 0.38 & 0.42 \\ 8 \mathrm{e}-03 & 0.264 & 0.25 & 0.27 & 0.33 & 0.38 & 0.42 \\ 9 \mathrm{e}-03 & 0.266 & 0.25 & 0.27 & 0.33 & 0.38 & 0.42 \\ 1 \mathrm{e}-02 & 0.268 & 0.25 & 0.27 & 0.33 & 0.38 & 0.42\end{array}$

The table of chemical compositions presents all the $y$ values available for a given $z$. For a set of parameters, the track files are identified specifying the mass of the desired model (in the range [0.30 1.10] $M_{\odot}\left(M_{\odot}=1.99 \cdot 10^{33} \mathrm{~g}\right.$ is the mass of the Sun), in steps of $\left.0.05 M_{\odot}\right)$, while the age (in the range [8.0 - 15.0] Gyr, in steps of $0.5 \mathrm{Gyr}$ ) is required for the isochrones.

Upon specification of the aforementioned parameters, the stellaR package can import data from CDS (via anonymous ftp) over an active Internet connection. The CDS data are stored in ASCII format and include a header with calculation metadata, such as the metallicity, the initial helium abundance, and the mixing-length. The import is done via a read.table() call, skipping the header of the files.

The following data objects can be downloaded from the database site:

- Stellar track: a stellar evolutionary track computed starting from Pre-Main Sequence (PMS) and ending at the onset of helium flash (for masses $M \geq 0.55 M_{\odot}$ ) or at the exhaustion of central hydrogen (for $0.30 M_{\odot} \leq M \leq 0.50 M_{\odot}$ ). The functions getTrk() and getTrkSet() can be used to access such data; they respectively return objects of classes "trk" and "trkset".

- Stellar ZAHB: Zero-Age Horizontal-Branch models. The function getZahb() can be used to access such data; it returns an object of class "zahb".

- HB models: computed from ZAHB to the onset of thermal pulses. The functions getHb() and getHbgrid() can be used to access such data; they respectively return objects of classes "hb" and "hbgrid".

- Stellar isochrones: computed in the age range [8.0 - 15.0] Gyr. The functions getIso() and getIsoSet() can be used to access such data; they respectively return objects of classes "iso" and "isoset".

Readers interested in details about the computation procedure are referred to Dell'Omodarme et al. (2012). The data gathered from CDS are organized into objects of appropriate classes. The package includes print and plot S3 methods for the classes "trk", "trkset", "zahb", "hb", "hbgrid", "iso", and "isoset".

As an example, we illustrate the recovering of the stellar track for a model of mass $M=0.80 M_{\odot}$, metallicity $z=0.001$, initial helium abundance $y=0.25$, mixing-length $\alpha_{\mathrm{ml}}=1.90$, $\alpha$-enhancement $[\alpha / \mathrm{Fe}]=0.0$.

$>\operatorname{track}<-\operatorname{getTrk}(\mathrm{m}=0.80, \mathrm{z}=0.001, \mathrm{y}=0.25, \mathrm{ml}=1.90$, afe $=0)$

$>$ track

Stellar track

Mass $=0.8$ Msun

$Z=0.001, Y=0.25$

Mixing length $=1.9$

$[$ alpha/Fe $]=0$

$>\operatorname{names}($ track)

[1] "mass" "z" "yl" "alpha.enh" "data"
$>$ class(track)

[1] "trk" "stellar"

The function getTrk() returns an object of class "trk", which is a list containing the track metadata, i.e. the star mass, the metallicity, the initial helium abundance, the mixing-length and the $\alpha$-enhancement, and the computed data in the data frame data. Track data contains the values of 15 variables:

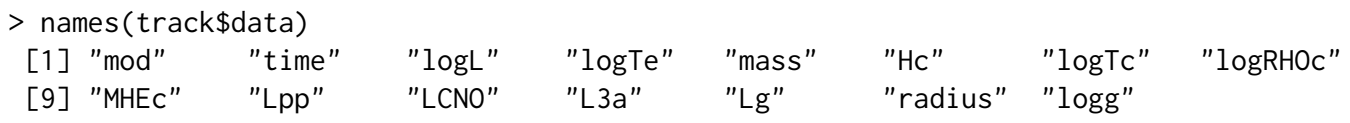

The included variables are: mod the progressive model number; time the logarithm of the stellar age (in $y r)$; logL the logarithm of the surface luminosity (in units of solar luminosity); logTe the logarithm of 
the effective temperature (in K); mass the stellar mass (in units of solar mass); Hc the central hydrogen abundance (after hydrogen exhaustion: central helium abundance); logTc the logarithm of the central temperature (in K); logRHOc the logarithm of the central density (in $\mathrm{g} / \mathrm{cm}^{3}$ ); MHEc the mass of the helium core (in units of solar mass); Lpp the luminosity of pp chain (in units of surface luminosity); LCNO the luminosity of CNO chain (in units of surface luminosity); L3a the luminosity of triple- $\alpha$ burning (in units of surface luminosity); Lg luminosity of the gravitational energy (in units of surface luminosity); radius the stellar radius (in units of solar radius); logg the logarithm of surface gravity (in $\mathrm{cm} / \mathrm{s}^{2}$ ).

Similarly the part of the track starting from ZAHB and ending at the onset of thermal pulses can be downloaded with the call:

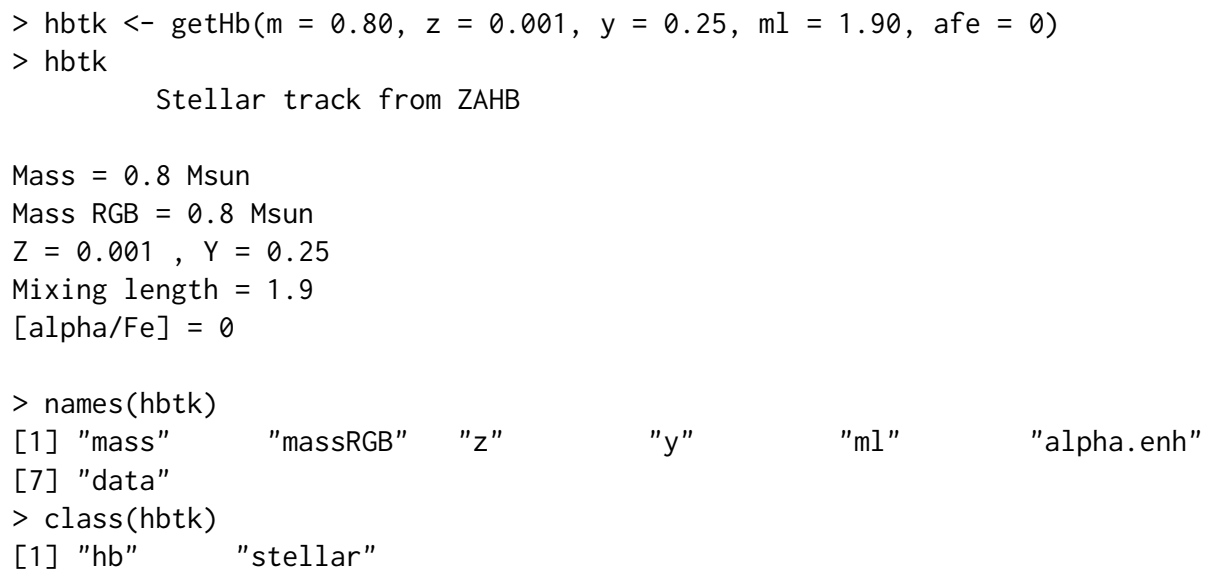

Function get $\mathrm{Hb}()$ returns an object of class "hb", which differs from an object of class "trk" only for the presence of the variable massRGB, i.e. the Red-Giant Branch (RGB) progenitor mass.

Usually a set of tracks with different mass and/or metallicity values are needed for computations. The package stellaR provides the function getTrkSet(), which can download a set of tracks with different values for mass, metallicity, initial helium abundance, mixing-length and $\alpha$-enhancement. As an example the whole set of masses (from 0.30 to $1.10 M_{\odot}$, in steps of $0.05 M_{\odot}$ ), for metallicity $z=0.001$, initial helium abundance $y=0.25$, mixing-length $\alpha_{\mathrm{ml}}=1.90$, and $\alpha$-enhancement $[\alpha / \mathrm{Fe}]=$ 0.0 can be downloaded as follows:

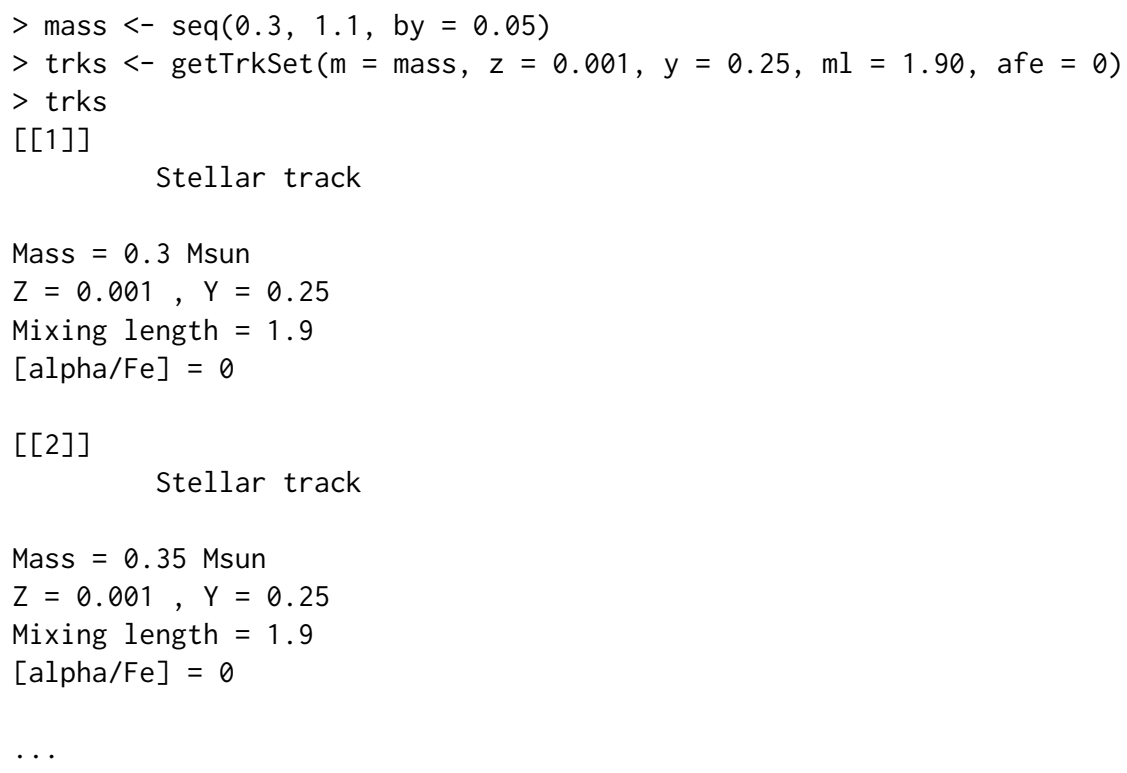

The function getTrkSet() returns an object of class "trkset", a list containing objects of class "trk". The track set can be displayed in the usual $\left(\log T_{\text {eff }}, \log L / L_{\odot}\right)$ plane by a call of the function plot():

$>\operatorname{plot}($ trks, 1 ty $=1: 2)$

The output of the function is shown in Figure 1. The plot is produced by a call to the function plotAstro(), which allows the user to customize several aspects of the plot, such as the axes labels, the number of minor ticks between two major ticks, the limits of the axes, the color and type of the lines (as in the example), the type of the plot (lines, points, both,...). 


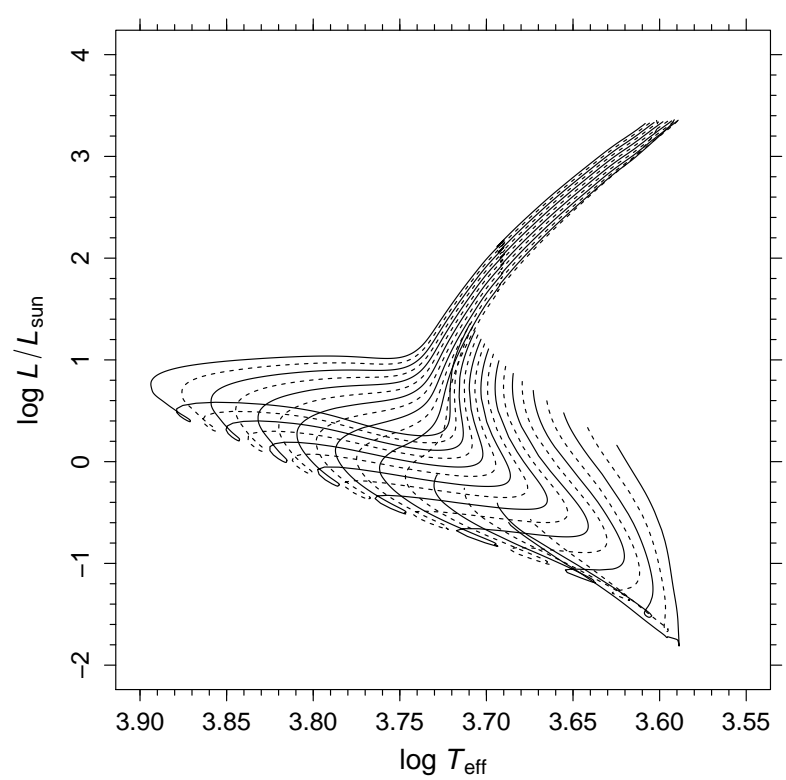

Figure 1: The evolutionary tracks for masses from $M=0.30 M_{\odot}$ to $M=1.10 M_{\odot}$ from PMS to He flash. The parameters of the calculations are: $z=0.001, y=0.25, \alpha_{\mathrm{ml}}=1.90,[\alpha / \mathrm{Fe}]=0.0$.

The use of the plot () function is further demonstrated in Figure 2, where, for $z=0.001, y=0.25$, $\alpha_{\mathrm{ml}}=1.90,[\alpha / \mathrm{Fe}]=0.0$, the evolutionary tracks for $M=0.80 M_{\odot}$ from PMS to He flash (black line) and from ZAHB to thermal pulses (green line) are displayed. The figure is obtained as follows:

$>\operatorname{plot}($ track)

$>\operatorname{plot}($ hbtk, add $=$ TRUE, col $=$ "green")

Apart from the plots discussed before, it is easy to display other relations between the computed variables. In the following example we get the data for two masses, namely 0.50 and $1.00 M_{\odot}$, and plot the trend of the radius (in units of solar radius) versus the logarithm of the age for the first 100 models. The resulting plot is displayed in Figure 3.

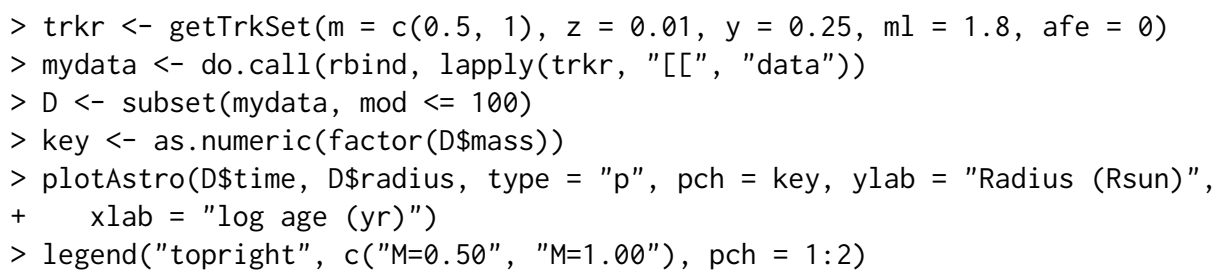

Isochrones can be obtained from CDS and plotted in a similar way. As an example, we get isochrones of 9 and 12 Gyr for $z=0.001, y=0.25, \alpha \mathrm{ml}=1.90,[\alpha / \mathrm{Fe}]=0.0$ :

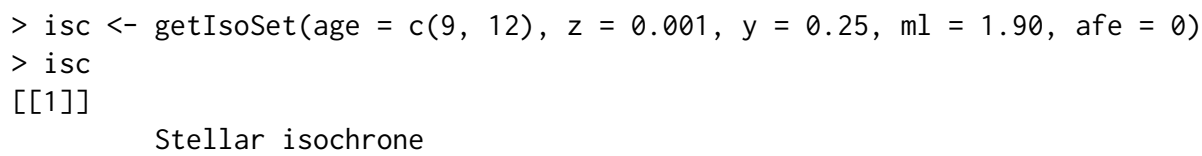




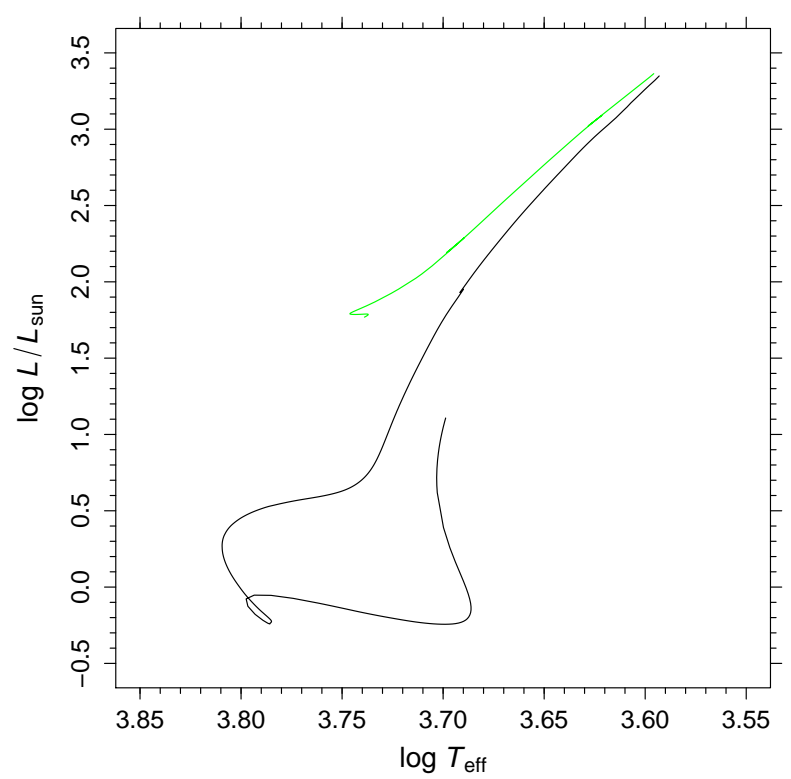

Figure 2: Black line: evolutionary tracks for mass $M=0.80 M_{\odot}, z=0.001, y=0.25, \alpha_{\mathrm{ml}}=1.90,[\alpha / \mathrm{Fe}]$ $=0.0$ from PMS to He flash. Green line: evolutionary track from ZAHB to thermal pulses.

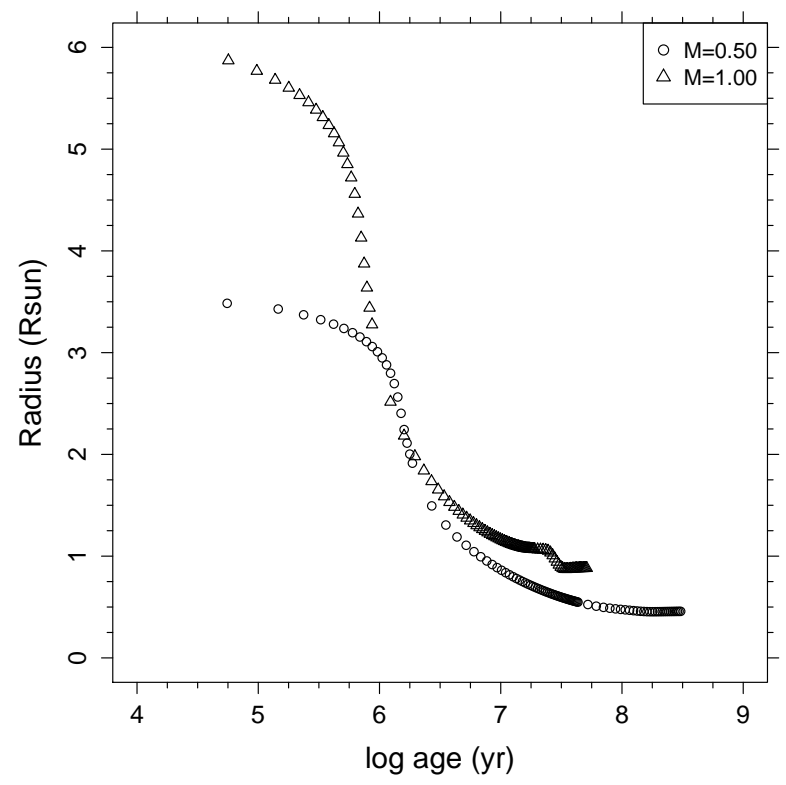

Figure 3: Radius versus the logarithm of the age for the first 100 models of two stars with different mass $\left(M=0.50 M_{\odot}\right.$ and $\left.M=1.00 M_{\odot}\right)$ and identical composition, $z=0.01, y=0.25, \alpha_{\mathrm{ml}}=1.8,[\alpha / \mathrm{Fe}]=$ 0.0 . 


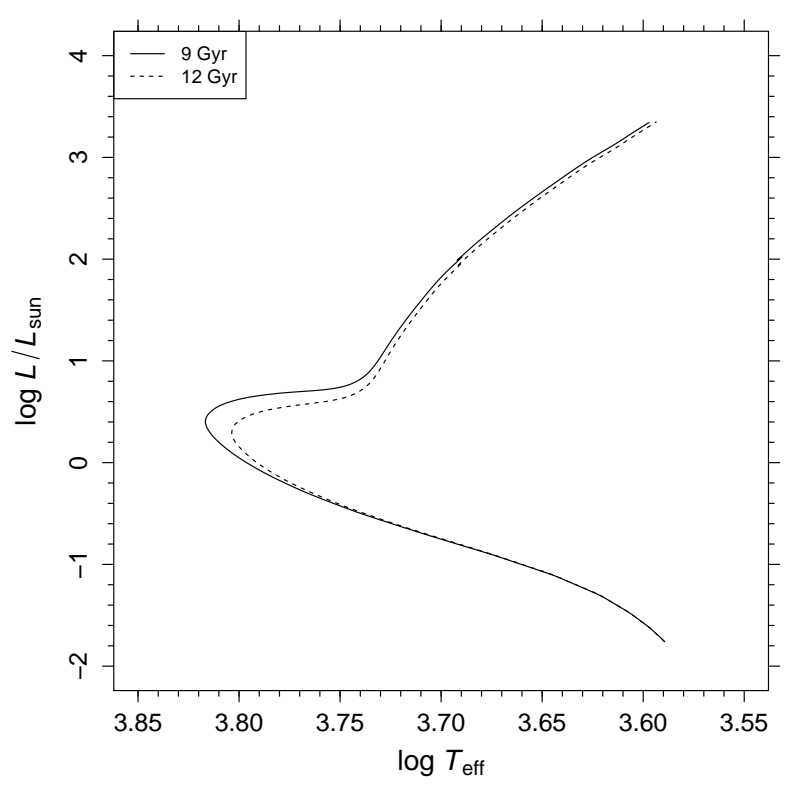

Figure 4: Isochrones in the theoretical plane for 9 and 12 Gyr. Calculations performed for $z=0.001, y$ $=0.25, \alpha_{\mathrm{ml}}=1.90,[\alpha / \mathrm{Fe}]=0.0$.

$\operatorname{attr}($, "class")

[1] "isoset" "stellar"

$>\operatorname{names}(\operatorname{isc}[[1]])$

[1] "age" "y" "ml" "alpha.enh" "data"

$>$ names(isc $[[1]] \$$ data)

[1] "logL" "logTe" "mass" "radius" "logg"

The function returns an object of class "isoset", a list containing objects of class "iso". The latter objects are lists containing metadata (age, metallicity, initial helium abundance, mixing-length, $\alpha$ enhanchment) and the data frame data, which contains the computed theoretical isochrones data. Figure 4 shows the set of isochrones plotted with the commands:

$>\operatorname{plot}($ isc, 1 ty $=1: 2)$

> legend("topleft", c("9 Gyr", "12 Gyr"), lty = 1:2)

\section{Tools for interpolating among data structures}

Even if the database provides a fine grid of models it is possible that the specific model needed for a comparison with observational data has not been calculated. To address this issue, the package stellaR includes tools for constructing new sets of isochrones. The simplest case is whenever one desires an isochrone for ages not included in the database, but for a combination of metallicity, initial helium abundance, mixing-length and $\alpha$-enhanchment existing in the database. The function makeIso() is able to compute the required isochrones by means of interpolation on a set of tracks. The interpolation can be performed for age in the range [7.0 - 15.0] Gyr. The user has the choice to explicitly provide to the function a set of tracks, previously downloaded from CDS, or to specify the required composition of the tracks to be downloaded for the interpolation. To show the usage of the function we use the object trks downloaded before to obtain an isochrone of age 9.7 Gyr:

$>$ iso.ip $<-$ makeIso $($ age $=9.7, \operatorname{tr}=$ trks $)$

$>$ iso.ip

Stellar isochrone

Age $=9.7 \mathrm{Gyr}$

$\mathrm{Z}=0.001, \mathrm{Y}=0.25$

Mixing length $=1.9$

$[\mathrm{alpha} / \mathrm{Fe}]=0$

The call produces a result identical to makeIso (age $=9.7, \mathrm{z}=0.001, \mathrm{y}=0.25, \mathrm{ml}=1.9$, afe $=0$ ); in the latter case the data are taken from CDS before the interpolation procedure. 
The interpolation technique is based upon the fact that all the tracks contain the same number of points by construction, and that a given point corresponds to the same evolutionary phase on all the tracks. We define $S(M)$ as the set of tracks to be used for interpolation, parametrized by the value of the mass $M$. Let $t_{i}(M)$ be the evolutionary time for the $i$ th point on the track of mass $M$, and $A$ be the age of the required isochrone. Let $k$ be the point on the track of lower mass of the set $S(M)$ for which $t_{k}(M) \geq A$. For each point $j \geq k$ on the tracks in $S(M)$, a linear interpolation in age of the values of mass, logarithm of the effective temperature and logarithm of the luminosity is performed among tracks. These points define the required isochrone. A potential problem of this simple procedure will occur whenever massive stars develop a convective core during the Main Sequence (MS). In this case, as shown for example in Mowlavi et al. (2012), the monotonic trend of the evolutionary time - that decreases with increasing stellar mass at the end of the MS - inverts at the middle of the MS. However the problem will be encountered only for early-time isochrones, for which the mass at the isochrone Turn-Off will be in the interval during which the convective core develops. The procedure outlined in this Section is adequate for construction of isochrones throughout the range allowed by the function.

\section{Track interpolation}

The package stellaR provides also a tool for performing a 3D interpolation on the database to construct a set of tracks for values of metallicity, initial helium abundance and mixing-length not included in the computations available at CDS. The function interpTrk() can be used for this procedure. A call to this function causes the download from CDS of the sets of tracks needed for the interpolation.

The new set of tracks is computed by means of a linear interpolation. The metallicity is logtransformed before the interpolation procedure. Let $T_{z, y, \alpha_{\mathrm{ml}}}(M)_{i}$ be the $i$ th point in the data set containing the evolutionary time, the effective temperature and the logarithm of surface luminosity for the track of mass $M$ and given composition. The interpolation algorithm proceeds as follows:

$$
\overbrace{T_{z, y, \alpha_{\mathrm{ml}}}(M)_{i}}^{8 \text { sets }} \rightarrow \overbrace{T_{z, y, *}(M)_{i}}^{4 \text { sets }} \rightarrow \overbrace{T_{z, *, *}(M)_{i}}^{2 \text { sets }} \rightarrow \overbrace{T_{*, *, *}(M)_{i}}^{1 \text { set }}
$$

The symbol $*$ means that interpolation occurred in the substituted variable. The selection of the set of tracks which enter in the interpolation is based upon the identification of the vertexes of the cell of the $\left(z, y, \alpha_{\mathrm{ml}}\right)$ space containing the point identified by the required parameters. Then, for all the 17 masses at the vertexes, the linear interpolation described above is performed. In the worst case scenario, whenever none of the supplied parameters values exists in the database, the interpolation requires $2^{3}=8$ sets of 17 masses. The algorithm is however able to reduce the dimensionality of the process if some of the variable values exist in the database.

As a demonstration, let us compute a set of tracks with mixing-length value $\alpha_{\mathrm{ml}}=1.74, z=0.002, y$ $=0.25,[\alpha / \mathrm{Fe}]=0.0$ :

$>$ ip.trk <- interpTrk $(z=0.002, y=0.25, \mathrm{ml}=1.74$, afe $=0)$

Since the values of $z$ and $y$ exist in the database, only an interpolation on the mixing-length value is performed by the function. The set of tracks can be used for isochrone construction, like a standard set of tracks:

$>$ ip.iso $<-$ makeIso (age $=12, \operatorname{tr}=$ ip.trk)

\section{Keypoints extraction}

Important stages in the evolution of a star are defined as "keypoints", e.g. hydrogen core exhaustion, Turn-Off luminosity, RGB tip luminosity. To simplify their extraction the package stellaR provides the function keypoints(), which operates on an object of class "trk" or "iso".

The function extracts from the data stored in objects of class "trk" the rows of the data frame relative to the following evolutionary stages:

1. ZAMS. Zero-Age Main-Sequence, defined as the point for which the central $\mathrm{H}$ abundance drops below $99 \%$ of its initial value.

2. TO. Turn-Off, defined as the point for which the effective temperature reaches its maximum value. If multiple lines satisfy the constraint, the values of all the rows are averaged.

3. BTO. Brighter Turn-Off, defined as the point for which the effective temperature drops below the temperature of the TO minus $100 \mathrm{~K}$. This point can not exist for low masses. Details on the advantages of this evolutionary point with respect to the TO can be found in Chaboyer et al. (1996). 
4. exHc. Central $\mathrm{H}$ exhaustion, defined as the point for which the central $\mathrm{H}$ abundance is zero. For low masses the point can coincide with TO. This is the last point of the tracks with mass lower or equal to $0.50 \mathrm{M} \odot$.

5. Heflash. Helium flash, the last point of the track for masses higher than $0.50 \mathrm{M}_{\odot}$.

When the function is called on an object of class "iso" it returns a data frame containing only TO and BTO phases.

In both cases the function inserts in the returned data frame the columns relative to mass (or age for isochrones), metallicity, initial helium abundance value, mixing-length, $\alpha$-enhancement, and evolutionary phase identifier.

As a demonstration we extract the TO and BTO points from the object isc generated in a previous example:

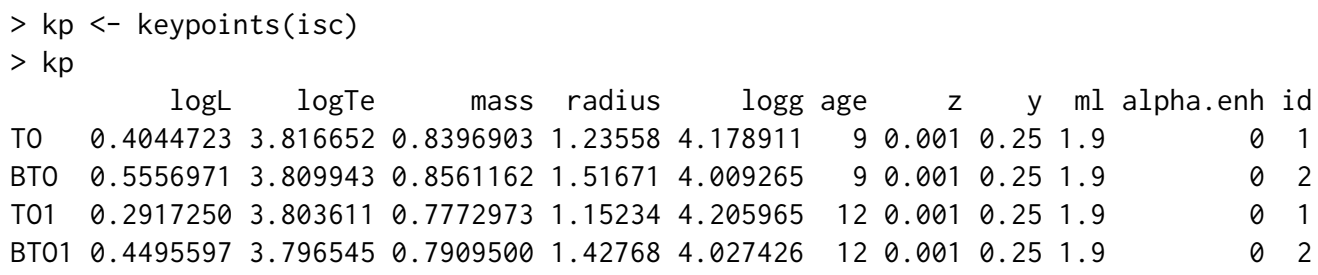

The points can be easily superimposed to the isochrones in the theoretical plane. The top panel of Figure 5 is obtained with the following commands:

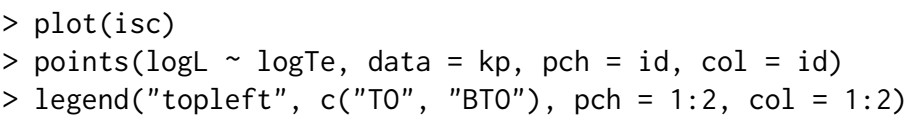

As a last example we extract a set of tracks for masses in the range [0.40 - 1.10] $M_{\odot}$ and three metallicity values $z=0.0001,0.001,0.01$ and we display the time of exhaustion of central hydrogen as a function of the mass of the star (bottom panel in Figure 5).

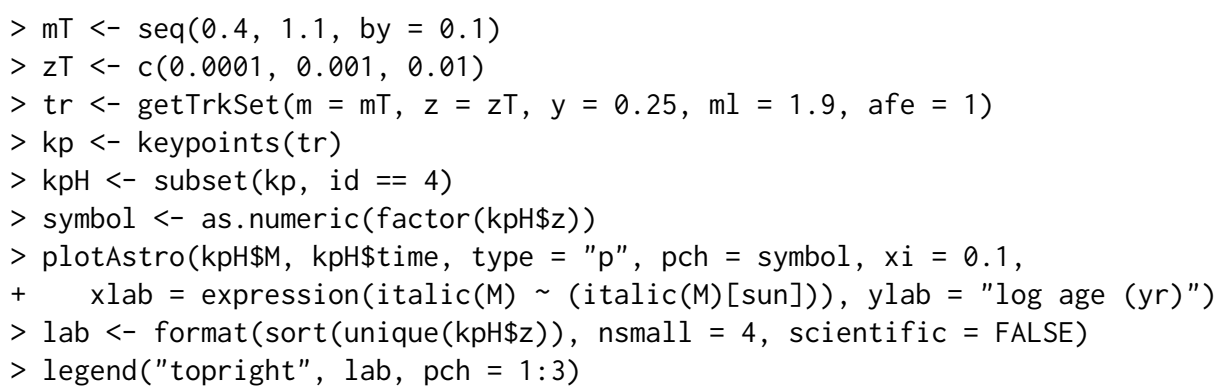

\section{Summary}

This paper demonstrated how the package stellaR can be useful to the astrophysical community as a tool to simplify the access to stellar tracks and isochrone calculations available on-line. A set of tools to access, manipulate and plot data are included in the package and their usage was shown. The interpolation functions included in the package can be used to safely produce tracks or isochrones at compositions not included in the database, without the need that users develop software on their own. A planned extension of the package is the modification of the algorithm of isochrone construction to make the calculation of isochrones of young ages feasible. This step can be useful in view of a possible extension of the Pisa database to higher masses, or to manipulation of data stored in other databases. In fact, while the package is currently developed for accessing data from the Pisa low-mass database, other public databases can be in principle accessed in the same way. This step is however complicated by the fact that no standard for the stellar model output exists in the astrophysical community, requiring the individual adaptation of the interface functions for each data set.

\section{Acknowledgments}

We are grateful to our anonymous referees for many stimulating suggestions that helped to clarify and improve the paper and the package. We thank Steve Shore for a careful reading of the manuscript and many useful suggestions. 

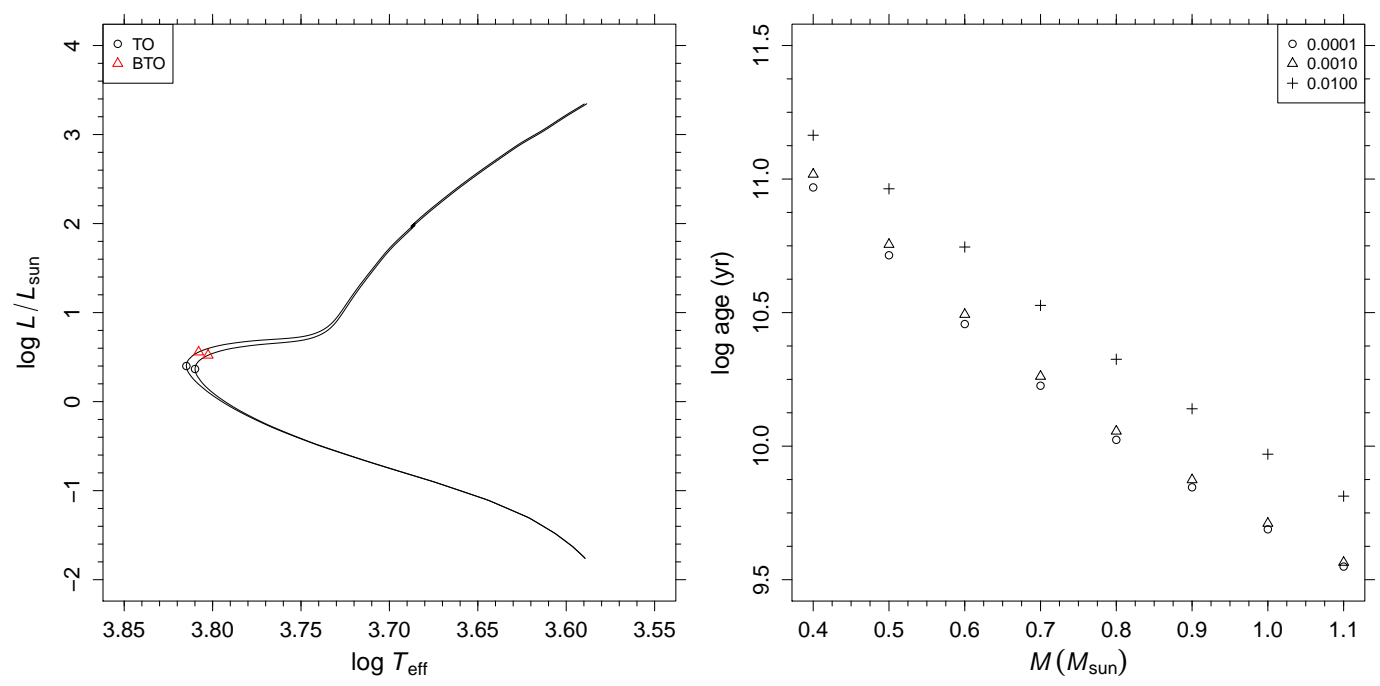

Figure 5: Top panel: same as Figure 4. The position of Turn-Off (TO) and Brighter TO (BTO) are shown. Bottom panel: time to exhaustion of central hydrogen as a function of the mass of the star. The symbols identify the values of the metallicity $z$ from 0.0001 to 0.01 .

\section{Bibliography}

G. Bertelli, L. Girardi, P. Marigo, and E. Nasi. Scaled solar tracks and isochrones in a large region of the $Z-Y$ plane. I. From the ZAMS to the TP-AGB end for 0.15-2.5 $M_{\odot}$ stars. Astronomy $\mathcal{E}$ Astrophysics, 484(3):815-830, 2008. [p108]

B. Chaboyer, P. Demarque, P. J. Kernan, L. M. Krauss, and A. Sarajedini. An accurate relative age estimator for globular clusters. Monthly Notices of the Royal Astronomical Society, 283(2):683-689, 1996. [p114]

M. Dell'Omodarme and G. Valle. stellaR: Stellar Evolution Tracks and Isochrones, 2012. URL http: //CRAN. R-project. org/package=stellaR. R package version 0.3-1. [p108]

M. Dell'Omodarme, G. Valle, S. Degl'Innocenti, and P. G. Prada Moroni. The Pisa Stellar Evolution Data Base for low-mass stars. Astronomy \& Astrophysics, 540:A26, 2012. [p108, 109]

A. Dotter, B. Chaboyer, D. Jevremović, V. Kostov, E. Baron, and J. W. Ferguson. The Dartmouth Stellar Evolution Database. Astrophysical Journal Supplement Series, 178(1):89-101, 2008. [p108]

N. Mowlavi, P. Eggenberger, G. Meynet, S. Ekström, C. Georgy, A. Maeder, C. Charbonnel, and L. Eyer. Stellar mass and age determinations. I. Grids of stellar models from $Z=0.006$ to 0.04 and $M=0.5$ to $3.5 M_{\odot}$. Astronomy E Astrophysics, 541:A41, 2012. [p114]

A. Pietrinferni, S. Cassisi, M. Salaris, and F. Castelli. A large stellar evolution database for population synthesis studies. II. Stellar models and isochrones for an $\alpha$-enhanced metal distribution. Astrophysical Journal, 642(2):797-812, 2006. [p108]

\section{Matteo Dell'Omodarme}

Dipartimento di Fisica "Enrico Fermi", Università di Pisa

Largo Pontecorvo 3, Pisa I-56127

Italy

mattdell@fastmail.fm

Giada Valle

Dipartimento di Fisica "Enrico Fermi", Università di Pisa

Largo Pontecorvo 3, Pisa I-56127

Italy

valle@df.unipi.it 